\section{International Scientific Journal Theoretical \& Applied Science}

p-ISSN: 2308-4944 (print) e-ISSN: 2409-0085 (online)

Year: 2015 Issue: 06 Volume: 26

Published: $30.06 .2015 \quad$ http://T-Science.org

SECTION 7. Mechanics and machine construction.
Ashirbek Zhakash

Docent, Candidate of Technical Sciences

Taraz State University named after M.Kh. Dulati,

Kazakhstan

\section{Adilgazi Amangalyevich Talasbaev}

student

Taraz State University named after M.Kh. Dulati,

Kazakhstan

Adilgazi@bk.ru

Aidana Raymova

student

Taraz State University named after M.Kh. Dulati,

Kazakhstan

\title{
SELF-SYNCHRONIZATION OF TWO ONE-MASSIN RESONANT VIBRATORS
}

Abstract: Discusses the possibility of self-synchronization of two one-massin resonant vibrator connected by an elastic connection. For research of self-synchronization of two resonant vibrators with the analytical characteristics of elastic ties a program was compiled in Delphi. The possibilities of existence in the system - the effect of self-synchronization are shown.

Key words: self-synchronisation, crank, resonant vibrocars, systems with one degree of freedom, sinfaza.

Language: Russian

Citation: Zhakash A, Talasbaev AA, Raymova A (2015) SELF-SYNCHRONIZATION OF TWO ONEMASSIN RESONANT VIBRATORS. ISJ Theoretical \& Applied Science 06 (26): 48-51.

Soi: http://s-o-i.org/1.1/TAS*06(26)11 Doi: crossef http://dx.doi.org/10.15863/TAS.2015.06.26.11

\section{УДК 531.1:514.1}

\section{САМОСИНХРОНИЗАЦИИ ДВУХ ОДНОМАССНЫХ РЕЗОНАНСНЫХ ВИБРОМАШИН}

Аннотация: Рассматривается возможность самосинхронизации двух одномассньх резонансньх вибромашин, связанных упругой связью. Для исследования самосинхронизации двух резонансных вибромашин с аналитическими характеристиками упругих связей была составлена программа на языке Delphi. Показаны возможности существования в системе эффекта самосинхронизации.

Ключевые слова: самосинхронизация, кривошип, резонансные вибромашины, системы с одной степенью свободы, синфаза.

Рассматривается возможность самосинхронизации двух одномассных резонансных вибромашин, связанных упругой связью [1]. Схема рассматриваемой системы представлена на рис. 1.

Будем полагать, что каждый блок представляет собой упругую систему с одной степенью свободы. В рассматриваемой системе возбуждение колебаний осуществляется эксцентриковыми вибраторами с упругими элементами в шатуне. Упругие восстанавливавшие характеристики основных упругих связей будем описывать соотношениями вида

$$
P_{0}\left(x_{i}\right)=k_{i} x_{i}+\beta_{i} x_{i}^{3} \quad(i=1,2)
$$

Тогда для расчетной схемы, представленной на рис.1., при сделанных выше предположениях, дифференциальные уравнения движения могут быть записаны в виде:

$$
\begin{gathered}
m_{1} \ddot{x}_{1}+\varepsilon_{1} \dot{x}_{1}+\left(k_{10}+k_{1}\right) x_{1}+\beta_{1} x_{1}^{3}+k_{12}\left(x_{1}-x_{2}\right)=k_{10} \rho \sin \varphi_{1} \\
m_{2} \ddot{x}_{2}+\varepsilon_{2} \dot{x}_{2}+\left(k_{20}+k_{2}\right) x_{2}+\beta_{2} x_{2}^{3}-k_{12}\left(x_{1}-x_{2}\right)=k_{20} \rho \sin \varphi_{2} \\
J_{1} \ddot{\varphi}_{1}+g_{1} \ddot{\varphi}_{1}=M_{10}-b_{1} \dot{\varphi}_{1}+k_{10} \rho\left(x_{1}-\rho \sin \varphi_{1}\right) \cos \varphi_{1} \\
J_{2} \ddot{\varphi}_{2}+g_{2} \ddot{\varphi}_{2}=M_{20}-b_{2} \dot{\varphi}_{2}+k_{20} \rho\left(x_{1}-\rho \sin \varphi_{2}\right) \cos \varphi_{2}
\end{gathered}
$$

ISPC Education \& Innovation, 
где $m_{1}, m_{2}$ - массы рабочих органов;

$J_{1}, J_{2}$ - моменты инерции приведенные к кривошипам;

$k_{10}, k_{20}$ - жесткости приводных упругих связей;

$\varphi_{1}, \varphi_{2}$ - углы поворота кривошипов;

$k_{12}$ - жесткость упругой связи, соединяющей рабочие органы; $x_{1}, x_{2}$ - перемещения центров массы рабочих органов;

$M_{10}-b_{1} \dot{\varphi}_{1}, M_{20}-b_{2} \dot{\varphi}_{2}$ моментные характеристики двигателей в рабочей области, приведенные к кривошипам;

$\rho$ - радиус кривошипа (эксцентриситета).

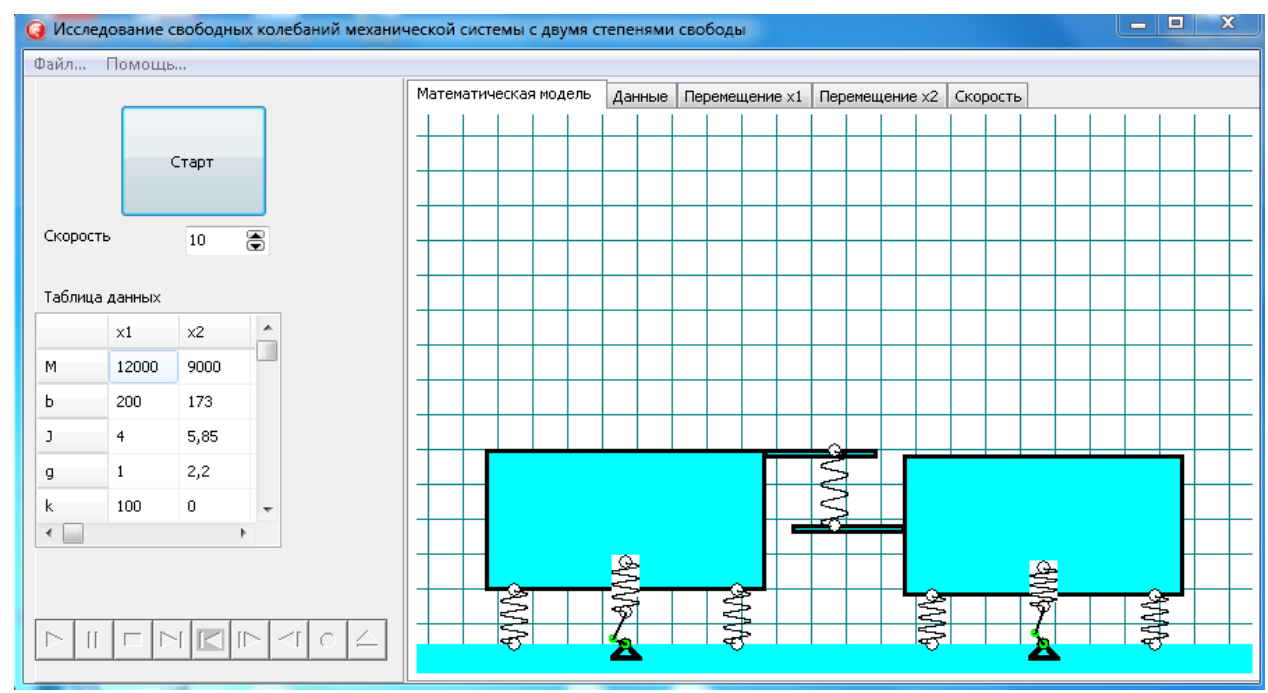

Рисунок 1 - Расчетная схема секционированной резонансной вибромашины.

Для исследования самосинхронизации двух резонансных вибромашин с аналитическими характеристиками упругих связей была составлена программа на языке Delphi [2]. Код программы указан в таблице. Программа позволяет визуально следить за работой системы где $k_{1}, k_{2}$ жесткости упругих связей.

В качестве конкретного объекта исследования была взята система со следующими значениями параметров:

$$
\begin{array}{ll}
m_{2}=7800 \kappa 2 ; & k_{1}=k_{2}=6.05 \cdot 10^{7} \mathrm{H} / \mathrm{M} ; \\
k_{10}=k_{20}=8 \cdot 10^{7} \mathrm{H} / \mathrm{M} ; & \beta_{1}=\beta_{2}=10^{11} \mathrm{H} / \mathrm{M}^{3} ; \\
\varepsilon_{1}=\varepsilon_{2}=8 \cdot 10^{5} \mathrm{c} \cdot \mathrm{H} / \mathrm{M} ; & \rho=0.015 \mathrm{M} ; \\
J_{1}=J_{2}=5.85 \mathrm{H} \cdot \mathrm{M} / \mathrm{c}^{2} ; & g_{1}=g_{2}=2.2 \mathrm{H} \cdot \mathrm{M} \cdot \mathrm{c} ; \\
M_{10}=M_{20}=10773.6 \mathrm{H} \cdot \mathrm{M} ; & b_{1}=b_{2}=173.86 \mathrm{H} \cdot \mathrm{M} \cdot \mathrm{c} ;
\end{array}
$$

Значения жесткости $\mathrm{k}_{12}$, массы $\mathrm{m}_{1}$ варьировались в следующих пределах: $k_{12}=10^{7} \mathrm{H} / \mathrm{M} \div 2 \cdot 10^{8} \mathrm{H} / \mathrm{M}_{\text {m }} \quad$ шагом $5 \cdot 10^{6} \mathrm{H} / \mathrm{M}$ $m_{1}=3800 \kappa 2 \div 7800 \kappa 2$ с шагом $500 \kappa 2$.

Это осуществлялось о целью оценки возможностей захватывания системы в режим самосинхронизации при изменении параметров. Варьирование значения $\mathrm{k}_{12}$ связано с тем, что при составлении уравнения движения предполагалось, что устанавливаемая на рабочие органы форма с (технологической нагрузкой или без неё) не обеспечивает превращения их в одно абсолютно твердое тело.

В связи с этим, в математическую модель была введена упругая связь с жесткостью $k_{12}$, которая может принимать любое значение, учитывая практическую невозможность ее контроля. 
Impact Factor ISRA (India) $\quad=\mathbf{1 . 3 4 4}$

Impact Factor ISI (Dubai, UAE) $=\mathbf{0 . 8 2 9}$

based on International Citation Report (ICR)

Impact Factor GIF (Australia) $\quad \mathbf{0} \mathbf{0 . 3 5 6}$
Impact Factor JIF

$=\mathbf{1 . 5 0 0}$

Impact Factor SIS (USA) $\quad=\mathbf{0 . 9 1 2}$

Impact Factor РИНЦ (Russia) $=\mathbf{0 . 1 7 9}$

Impact Factor ESJI (KZ) $\quad=1.042$
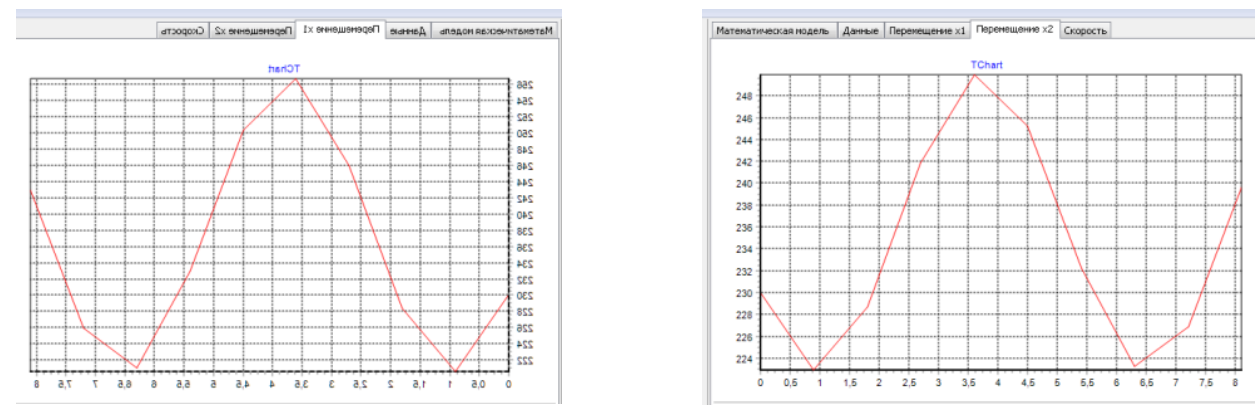

Рисунок 2 - Перемещение вибромашин.

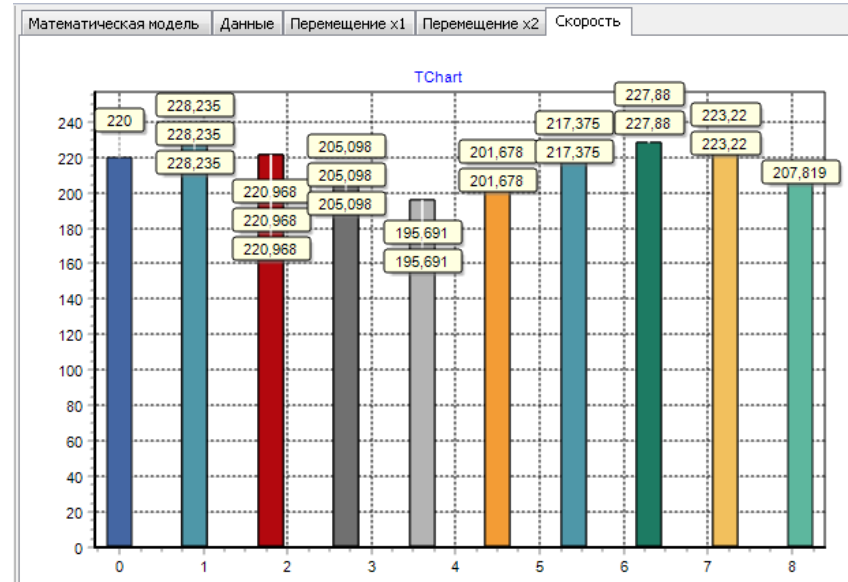

Рисунок 3 - Распределение скоростей.

Delphi code

\section{//////////////////////////////////////////////}

Kvadcolor1(x0+90,y0+75,20,30,clwhite);

$\mathrm{x} 10:=350+100 ; \mathrm{y} 10:=380$;

$\mathrm{x} 11:=\mathrm{x} 10 ; \mathrm{y} 11:=\mathrm{y} 0+80-20$;

image1.Canvas.Pen.Width:=3;

bekitpe(x10,y10,3);

shzel(trunc(x10), trunc $(y 10))$;

$\mathrm{x}:=\operatorname{Round}(\mathrm{x} 10+10 * \operatorname{Cos}(\mathrm{t} / 1.2)) ; \quad / /$ параметрическое уравнение для $\mathrm{x}$

$\mathrm{y}:=\operatorname{Round}(\mathrm{y} 10-10 * \operatorname{Sin}(\mathrm{t} / 1.2)) ; \quad$ //параметрическое

уравнение для у

image 1.Canvas.MoveTo(trunc(x10), $\quad \operatorname{trunc}(\mathrm{y} 10))$;

//центр окружности

image1.Canvas.LineTo(trunc $(\mathrm{X}), \quad \operatorname{trunc}(\mathrm{Y}))$;

//координаты х,у (докуда рисовать линию)

shzel (trunc $(\mathrm{x}), \operatorname{trunc}(\mathrm{y}))$;

$v x:=(x-x 11) / \operatorname{sqrt}(\operatorname{sqr}(x-x 11)+\operatorname{sqr}(y-y 11))$;

vy: $=(\mathrm{y}-\mathrm{y} 11) / \operatorname{sqrt}(\operatorname{sqr}(\mathrm{x}-\mathrm{x} 11)+\operatorname{sqr}(\mathrm{y}-\mathrm{y} 11))$;

$\mathrm{x} 12:=\mathrm{x} 11$;

$\mathrm{y} 12:=y-\operatorname{trunc}(20 * \mathrm{vy})$

image1.Canvas.MoveTo(trunc(x), $\operatorname{trunc}(\mathrm{y}))$;

image 1.Canvas.LineTo(trunc(x12), trunc(y12));

image1.Canvas.Pen.Width:=1;

pryz11(x0+100,y0+80,x12,y12);

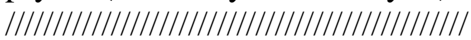

\section{////////////////////////////////////////////}

Kvadcolor1(x1+90,y1+75,20,30,clwhite);

$\mathrm{x} 10:=\mathrm{x} 1+100 ; \mathrm{y} 10:=380$;

$\mathrm{x} 11:=\mathrm{x} 10 ; \mathrm{y} 11:=\mathrm{y} 0+80-20$;

image1.Canvas.Pen.Width:=3;

bekitpe(x10,y10,3);

shzel(trunc (x10), trunc (y10));

$\mathrm{x}:=\operatorname{Round}(\mathrm{x} 10+10 * \operatorname{Cos}(\mathrm{t})) ; \quad$ //параметрическое

уравнение для $\mathrm{x}$

$\mathrm{y}:=\operatorname{Round}(\mathrm{y} 10-10 * \operatorname{Sin}(\mathrm{t})) ; \quad$ //параметрическое

уравнение для у

image 1.Canvas.MoveTo(trunc(x10), $\quad \operatorname{trunc}(\mathrm{y} 10))$;

//центр окружности

image1.Canvas.LineTo(trunc(X), $\quad \operatorname{trunc}(\mathrm{Y}))$;

//координаты х,у (докуда рисовать линию)

$\operatorname{shzel}(\operatorname{trunc}(\mathrm{x}), \operatorname{trunc}(\mathrm{y}))$;

$v x:=(x-x 11) / \operatorname{sqrt}(\operatorname{sqr}(x-x 11)+\operatorname{sqr}(y-y 11))$;

vy:=(y-y11)/sqrt(sqr(x-x11)+sqr(y-y11));

$\mathrm{x} 12:=\mathrm{x} 11$;

$\mathrm{y} 12:=y-\operatorname{trunc}(20 * \mathrm{vy})$;

image 1.Canvas.MoveTo(trunc $(\mathrm{x}), \operatorname{trunc}(\mathrm{y}))$;

image1.Canvas.LineTo(trunc(x12), trunc(y12));

image1.Canvas.Pen.Width:=1;

pryz11 $(\mathrm{x} 1+100, \mathrm{y} 1+80, \mathrm{x} 12, \mathrm{y} 12)$;

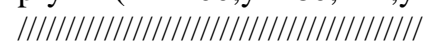


Impact Factor ISRA (India) $\quad=\mathbf{1 . 3 4 4}$

Impact Factor ISI (Dubai, UAE) $=\mathbf{0 . 8 2 9}$

based on International Citation Report (ICR)

Impact Factor GIF (Australia) $\quad \mathbf{0} \mathbf{0 . 3 5 6}$ $\begin{array}{lr}\text { Impact Factor JIF } & =\mathbf{1 . 5 0 0} \\ \text { Impact Factor SIS (USA) } & =\mathbf{0 . 9 1 2} \\ \text { Impact Factor PИНЦ (Russia) } & =\mathbf{0 . 1 7 9} \\ \text { Impact Factor ESJI (KZ) } & =\mathbf{1 . 0 4 2}\end{array}$

стремится к синфазному (рис.2). Этот факт следует учитывать при практическом использовании эффекта самосинхронизации в резонансных машинах, особенно в тяжелых машинах технологического назначения.

\section{References:}

1. Kryukov BM (1978) Dinamika sushchestvenno - nelineynykh sistem Mashinostroienie. Moscow, 1978.

2. Arkhangel'skiy AY (1999) Razrabotka prikladnykh programm dlya Windows V Delphi 5,- Moscow, 1999.

3. Gyuygens K (1970) Tri memuara po mekhanike. Perev. s angl., Fiz-matgiz, Moscow, 1970.

4. Blekhman II (1973) Samosinkhronizatsiya vibratorov nekotorykh vibratsionnykh mashin. Inzhenernyy sb., 1973, - pp. 16.

5. Gauze GF, Vitt AA (1934) O periodicheskikh kolebaniyakh chislennosti populyatsii. Izd-vo AN, ser. 7, 1934.
6. Tsaplin SA (1953) Viborudarnye mekhanizmy Avtotransizd., Moscow, 1953.

7. Blekhman II (1971) Sinkhronizatsiya dinamicheskikh sistem. Moscow, 896 p.

8. Lyapunov AM (1980) Obshchaya zadacha ob ustoychivosti dvizheniya. -Moscow. Gostekhizdat, $471 \mathrm{p}$.

9. Malkin IG (1950) Nekotorye zadachi teorii nelineynykh kolebaniy. Moscow. Gostekhizdat, $148 \mathrm{p}$.

10. Blekhman II (1978) Sinkhronizatsiya v tekhnike i prirode. -Nauka, $34 \mathrm{p}$. 